\title{
Tipología y funcionalidades de las aplicaciones móviles para mayores. A un tap del envejecimiento activo
}

institucional.us.es /ambitos/

\author{
Xabier Martínez-Rolán \\ Universidade de Vigo \\ xabier.rolan@uvigo.es \\ Teresa Piñero-Otero \\ Universidade da Coruña \\ teresa.pineiro@udc.es
}

English Version: Typology and functionality of the mobile applications for seniors. One tap away from activing aging

\section{Resumen}

En una sociedad cada vez más envejecida las nuevas tecnologías constituyen una oportunidad para mejorar la calidad de vida de las personas mayores. Dentro de este contexto cobran relevancia los smartphones en tanto que su penetración, portabilidad y accesibilidad favorecen el envejecimiento activo. El presente artículo ha tenido por objeto efectuar una aproximación a la oferta de aplicaciones móviles específica para seniors. El estudio efectuado permitió constatar la sencillez, accesibilidad y diseño ergonómico de estas aplicaciones, unas características que tendrán un papel determinante en las aplicaciones de comunicación interpersonal e

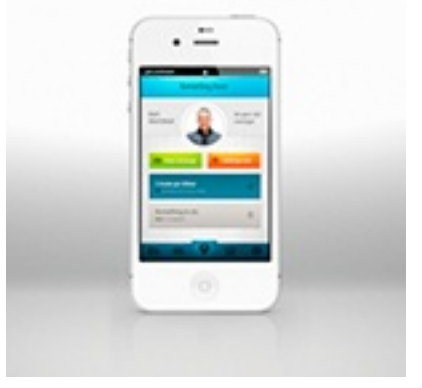
interacción social.

\section{Palabras clave}

APPs, smartphone, mayores, interacción social, envejecimiento activo.

Abstract

In an increasingly aging society, new technologies provide an opportunity to improve the quality of life of older people. Within this context smartphones become relevant because of its penetration, portability and accessibility

for an active aging. This article makes an approach to the specific mobile applications for seniors. The study reveals the simplicity, accessibility and ergonomic design of these applications, characteristics with a key role on interpersonal communication and social interaction.

\section{Keywords}

APPs, Smartphone, Seniors, social interaction, active aging.

\section{INTRODUCCIÓN}

El envejecimiento demográfico constituye una de las características de la sociedad actual. Por vez primera, en el siglo XXI el conjunto de población en edad madura ha logrado equipararse al joven. El incremento de la esperanza de vida y de su calidad han propiciado una dilación de la etapa adulta hasta edades avanzadas, lo que hace precisa una redefinición de la vejez y de sus fronteras (Parales y Dulce Ruiz, 2002; Lehr, 1980).

Hoy los individuos que viven su madurez ya no son viejos desde una perspectiva beauvoriana (Beauvoir, 1983). Tampoco se puede hablar de un período etario concreto que delimite la vejez (De Andrés y Maestro; 2014; Giró, 2004).

Si bien su construcción social suele basarse en criterios económicos (Fericla, 2002; De Andrés y Maestro, 
2014), resulta contradictorio que en un momento en el que la esperanza de vida supera los 80 años, y con ella la etapa activa del individuo, se continúe señalando la jubilación como inicio del declive vital (Aldana, Fonseca y García Gómez, 2013).

En este contexto cobra relevancia el "envejecimiento activo" entendido como el proceso de optimización de las oportunidades de salud, participación y seguridad para la mejora de la calidad de vida a medida que las personas envejecen (OMS, 2002). Se trata de un proceso encaminado a la construcción de una sociedad para todas las edades donde se eliminan las barreras para la independencia de las personas de edad y se fomenta una participación social plena (Sánchez Martínez y Hatton-Yeo, 2012). Un proceso en el que cobran relevancia las TICs en tanto que constituyen una herramienta clave para una sociedad más cohesionada e inclusiva (Plaza et altres, 2011).

Las investigaciones desarrolladas sobre las TICs en el segmento poblacional de mayor edad, especialmente en lo que respecta a los entornos de movilidad, convergen en un mismo aspecto: la relevancia de que las personas mayoras puedan estar y vivir en sus hogares de forma independiente el mayor tiempo posible (Plaza et altres, 2011). Este aspecto ha orientado el diseño de las aplicaciones móviles para seniors, en sus múltiples manifestaciones, objeto de estudio del presente trabajo.

\section{TICS PARA LA INCLUSIÓN SOCIAL}

El proceso de adopción de las nuevas tecnologías no ha sido generacionalmente neutro. Las personas de edad presentan los menores índices de adopción de nuevas tecnologías como Internet (Cotten, 2011; File, 2013; Hsiyi et altres, 2014). En el ámbito español tres de cada cuatro habitantes han accedido a Internet, un acceso que solamente ha sido efectuado por el $25 \%$ de los mayores de 65 años (INE, 2014). Una diferencia que se acentúa de atender a la conexión desde dispositivos móviles.

Esta brecha digital, basada en la edad, suele alimentar una serie de prejuicios sobre el acceso y uso las TICs por los mayores (Martínez, Cabecinhas y Loscertales, 2011). Con frecuencia los alfabetización digital senior deben combatir creencias, como la carencia de habilidades para el manejo de dispositivos tecnológicos, o el rechazo de los mismos (Abascal y Civit, 2001).

En términos de inclusión digital los mayores suelen quedar relegados. La alfabetización digital de este grupo etario debe hacer frente al acceso y aprendizaje TIC teniendo en cuenta aspectos como una merma en sus capacidades motoras y cognitivas, así como una mayor presencia de otra tipología de discapacidades (Hsi-yi et altres, 2014; Seglers, Van Boxel y Jolles, 2009).

El éxito de la e-inclusión en la franja de personas de mayor edad reside tanto en las políticas de alfabetización digital como en el desarrollo de dispositivos y software adecuado para su acceso y apropiación por la población de mayor edad (Werner, 2011). En este sentido se habla "Gerotechnology" para referirse a las TIC diseñadas basándose en las necesidades de una sociedad cada vez más envejecida (Plaza et altres, 2011).

La utilización de las TICs durante la etapa de la vejez presenta grandes potencialidades para este grupo etario (Charnes, Parks y Sabel, 2001). La intervención en esta línea posibilita la conexión de los mayores con la actualidad de su entorno, fomentando su inclusión social y la consideración de la vejez como otra etapa de enriquecimiento personal (Aguado, Pascual y Fombona, 2013; Plaza et altres, 2011). Asimismo las TICs pueden ayudar a las personas de edad a mejorar su calidad de vida y el establecimiento de sus lazos sociales (Cornejo, Tentori, Favela, 2013).

Durante la vejez suelen limitarse las interacciones sociales. La pérdida de grupos de pertenencia (como el laboral) y las dificultades de movilidad u otro tipo de afecciones asociadas a esta etapa pueden suponer barreras para la interacción con su entorno. En esta línea, el uso de determinados dispositivos tecnológicos y aplicaciones permite a los mayores mantener contacto, especialmente con la familia, y estrechar relaciones sociales (Agudo, Pascual y Fombona, 2013; Martínez Cabecinhas y Loscertales, 2011).

Frente a la "crisis de comunidad" augurada por Putnam (1995) el uso de las TICs permite reducir el aislamiento 
y soledad, incrementar la motivación y autoestima, desarrollar determinadas habilidades y mejorar su calidad de vida (Hsin-yi et altres, 2014; Amichai-Hamburger y Schenider, 2014). Aunque la tecnología per se no es determinante, las decisiones relacionadas con ésta permiten diversos tipos de interacción social (Hampton y Gupta, 2008).

Si se parte de la definición de gerotecnología como el estudio de las TICs para garantizar la salud, una participación social plena y la vida independiente (Plaza et altres, 2011; Harrington y Harrington, 2000), se pueden considerar que los dispositivos móviles y sus aplicaciones constituyen ejemplos paradigmáticos de esta tendencia.

La elevada penetración alcanzada por smartphones e Internet móvil ha propiciado el desarrollo de aplicaciones para seniors. Dichas aplicaciones pretenden dar respuesta a las necesidades que presenta este grupo etario basándose en las propias capacidades del terminal como dispositivo multimedia, donde convergen características del medio digital con otras potencialidades como portabilidad, conexión always \& everywhere, geolocalización, detección de movimiento, tactilidad, etc.

\section{DEL TELÉFONO AL DISPOSITIVO MULTIMEDIA}

Los terminales móviles han pasado a formar parte del equipamiento tecnológico personal de los mayores (Kurniawan, 2007). El 61\% de las personas con más de 65 años de países desarrollados son usuarios de teléfono móvil (Fondo de Población de Naciones Unidas, 2012). Una penetración que duplica a la de Internet en este segmento de población y que autores como Plaza et altres (2011) explican con la percepción de seguridad, de apoyo o de contacto personal asociadas al uso del teléfono móvil (Oksman, 2006; Conci, 2007; Ling, 2008).

Las necesidades y expectativas de las personas de edad respecto a la tecnología móvil constituye la base de su adopción (Phiriyapokanon, 2011). No obstante, pese a que los mayores perciben las oportunidades de la telefonía móvil para su calidad de vida, con frecuencia la rechazan por la complejidad y dificultad de su uso (Czaja y Lee, 2007).

El proceso de natural de envejecimiento conlleva una serie de cambios degenerativos como disminución de la agudeza visual, pérdida auditiva, dificultades psicomotrices o una menor capacidad de atención y memoria (Phiriyapokanon, 2011). En esta línea Kurniawan (2007) identificó hasta cuatro problemas de accesibilidad de los terminales convencionales para los mayores: tamaño y funcionalidad de botones, complejidad de menús, dimensiones reducidas y tamaño de texto. Unos problemas que han sido superados con la irrupción de los smartphones (Zhou et altres, 2014)..

El lanzamiento del iPhone, en 2007 supuso un cambio radical tanto en la concepción de los terminales telefónicos como del mobile computing. Más que teléfonos es preciso hablar de pantallas, en las que convergen diversas funcionalidades y servicios, en su mayoría accesibles a través de APPs. Estas aplicaciones permiten la personalización del terminal móvil y, por tanto, su adaptación a las necesidades y capacidades de cada individuo (Coelho et altres, 2012; Phiriyapokanon, 2011; Czaja y Lee, 2007).

La investigación sobre envejecimiento y tecnologías móviles llevada a cabo en las últimas décadas se ha centrado en el estudio de cómo estos dispositivos y aplicaciones pueden ayudar a las personas de edad a adquirir las habilidades precisas para su desarrollo personal y social (Hsin-yi et altres, 2013).

En este sentido se pueden señalar diversas aproximaciones que versan sobre el desarrollo de APPs (para smartphones y tablets) adaptadas a las necesidades específicas de los ciudadanos senior. Las más numerosas, aquellas que se analizan las aplicaciones vinculadas al cuidado de la salud como las de Charnes, 2014, Gao y Koronios (2010), Plaza et altres (2010) y Blake (2008) sobre las APPs de salud en su sentido más amplio; la de Yu, Li y Gagnon (2009) sobre información de salud, de McGree-Lennon (2008) sobre sistemas de cuidado en el hogar.

Otros estudios, como el de Pedell et altres (2013) o el de Drobics y Smith (2014) se centran en las aplicaciones 
de casual games tanto para paliar el envejecimiento cognitivo y ejercitar algunas habilidades psicomotrices, como para entretener.

En lo que respecta a las necesidades sociales el análisis de las aplicaciones diseñadas para la interacción de los mayores con su entorno, función básica del terminal telefónico, apenas ha sido tratado. Si bien se pueden señalar dos aproximaciones, desde una perspectiva tecnológica, la de Williams, Ahamed y Chu (2014) y la de Burkahard y Koch (2012) que versan sobre el diseño, desarrollo y características de sendas aplicaciones para la interacción social de los ciudadanos de mayor edad.

Pese a constituir una de las perspectivas menos frecuentes dentro de la investigación sobre aplicaciones móviles para seniors, la relevancia de las APPs comunicativas y sociales es un hecho.

La interacción con su entorno supone una de las principales motivaciones para la adopción de la telefonía móvil por las personas de edad e, inclusive, la causante de la misma (Plaza et altres, 2011). La interacción social limita el aislamiento que afecta este grupo etario y permite desarrollar-mantener relaciones de amistad, estrechar lazos con la familia o favorecen el sentimiento de pertenencia. Asimismo las aplicaciones sociales para mayores estimulan la frecuencia de las interacciones sociales así como la interdependencia entre mayores y jóvenes (abuelos y nietos), dos recomendaciones de la OMS (2002) en la consecución de una sociedad para todas las edades.

\section{COMUNICACIÓN PERSONAL Y REDES SOCIALES}

El establecimiento de redes sociales sólidas repercute positivamente en la calidad de vida de las personas mayores, en su sentido más amplio. El uso de las plataformas de gestión de redes sociales online por los "navegantes plateados" estimula la consolidación de estos lazos personales al facilitar los intercambios entre nodos. Además de la inclusión y de la participación, la adopción de estos gestores 2.0 por las franjas de población de mayor edad, conllevan también un mayor grado de satisfacción social (Vilte et altres, 2013; Bell et altres, 2013).

A la hora de fortalecer sus relaciones sociales los mayores suelen concentrar sus mayores esfuerzos en las redes familiares (Bell et altres, 2013; Cagley y Lee, 2009). La posibilidad de conectarse con familiares alejados o geográficamente dispersos supone una importante motivación para la adopción, por parte de las personas de edad, de nuevas tecnologías y aplicaciones online (Vilte et altres, 2013). En esta línea Cornajo, Tentori y Fabela (2013) destacan el valor del contenido compartido en las plataformas sociales en el enriquecimiento de los encuentros y relaciones familiares.

Si bien las plataformas sociales fueron concebidas para usuarios jóvenes (Vilte et altres, 2013), cuestión que las ha convertido en ejemplos paradigmáticos de la brecha digital, los usuarios de mayor edad han comenzado a hacerse un lugar en éstas (Bell et altres, 2013).

A comienzos del año 2014, un estudio del Pew Research Centre (2014) destacaba el incremento de usuarios senior entre los perfiles de Facebook, situándose en torno al $50 \%$ de los internautas estadounidenses para dicha edad.

Las personas mayores han incrementado su participación en redes sociales, sin embargo este número continúa siendo demasiado bajo. De los 20 Millones de perfiles de Facebook España, solamente un 3,5\% (700.000 perfiles) corresponden con usuarios de 65 años o más (FacebookAds, 2014). Como consecuencia, un gran parte de los españoles de mayor edad se quedan al margen de los social media para el refuerzo de redes de relaciones, con el consecuente riesgo de aislamiento social (Cornejo, Tentori y Favela, 2013)

Si se parte de que las plataformas de social media pueden incrementar el engagement social (Valenzuela, Park y Knee, 2008 o Zhang, Jiang y Carroll, 2011), el uso de los servicios móviles va a contar con un destacado papel en la mejora de la vida en comunidad (Zhang, Jiang y Carrol, 2011), especialmente de la inclusión de las personas mayores. 


\section{MATERIALES Y MÉTODOS}

El presente trabajo ha tenido el propósito de estudiar las aplicaciones móviles diseñadas para satisfacer las necesidades de los "usuarios plateados", especialmente de aquellas relacionadas con la comunicación e interacción social.

En esta línea se han planteado dos objetivos principales: $(01)$ conocer la oferta de APPs para seniors desde la perspectiva del tipo de apoyo prestado y (O2) analizar la tipología y principales características de las APPs que dan apoyo a las relaciones sociales y participación de este grupo.

Para la consecución de dichos objetivos se ha desarrollado un estudio exploratorio, en dos etapas, de las APPs para smartphone disponibles en iTunes y Google Play, plataformas de los dos sistemas operativos móviles con mayor penetración (iOS y Android).

Pese a que, en la línea de Conde, García-Peñalbo y Matellán-Olivera (2014), se considera que un repositorio para seniors potenciaría la accesibilidad a estas aplicaciones, las plataformas de Mac y Google continúan siendo las principales proveedoras de APPs desde el smartphone.

A partir de la aplicación de estas plataformas para iPhone y Smartphone Android, se efectuó una búsqueda de aplicaciones con términos clave (en español e inglés) vinculados a la vejez: senior, elderly, mayores, old people, ancianos, etc.

Esta búsqueda, efectuada durante la primera semana de octubrede 2014, tuvo como resultado un total de 187 APPs para iPhone y 96 para Android, una vez eliminadas duplicidades y aquellas aplicaciones cuyo target no son específicamente las personas mayores (o su red personal).

A partir del análisis de contenidos de la descripción, funcionalidades y demandas de acceso a funciones del terminal en las aplicaciones para senior se efectuó una categorización de la oferta de APPs atendiendo a su finalidad.

En una segunda etapa, se seleccionaron aquellas aplicaciones vinculadas a las necesidades de interación de los mayores. Un conjunto de aplciaciones cuya tipología características y funcionalidades requirieron un análisis más pormenorizado.

\section{CLASIFICACIÓN DE LAS APPS PARA SENIORS. UNA PROPUESTA}

Desde la primera aproximación a la muestra se puso de relevancia que, si bien el diseño y funcionalidades de dichas APPS pretende dar respuesta a las necesidades de las personas de edad, el target de dichas aplicaciones no siempre son estas. De hecho se puede señalar un núcleo de APPs destinadas a la familia y a los profesionales de la salud, para que puedan seguir los progesos e implicarse en el cuidado de sus mayores. Este tipo de aplicaciones se diferencian del resto por contar con un diseño estándar, con una organización compleja, que contrasta con la simiplicidad y ergonomía de las APPs para usuarios plateados.

En cualquier caso el criterio fijado para la categorizacion de las aplicaciones para seniors no ha sido el público objetivo sino la finalidad de las mismas. En función de este criterio se ha establecido una clasificación de las APPs en torno a seis ítems: (1) accesibilidad, (2) salud, (3) seguridad, (4) vida cotidiana, (5) entretenimiento y (6) comunicación pesonal.

\subsection{Accesibilidad}

Una de las principales oportunidades de los smartphones para las personas de edad es su posibilidad de personalización a través de la adaptación del terminal a las necesidades y capacidades del usuario. En este sentido una de las tipologías de APPs más frecuente en la muestra (un total de 51 unidades que constituyen e $18 \%$ de la muestra) se orienta a la conversión del teléfono móvil en un dispositivo accesible o a la utilización del 
mismo para mejorar la accesibilidad de los mayores.

Al primer tipo, relativo a la accesibilidad del móvil, pertecen APPs ccomo WisePhone o Big Launcher cuyo permite un acceso ergonómico a las funcionalidades del dispositivo adaptado a las capacidades físicas y cognitivas de las personas de edad. Se trata de una tipología de APPs más habituales en la oferta para dispositivos Android dadas las mayores posibilidades de accesibilidad de los terminales iPhone.

En el segundo grupo, las accesibilidad con el móvil, se incluyen todas aplicaciones destinadas a mejorar la accesibilidad de los mayores como las APPs destinadas a incrementar la visibilidad y legibilidad, como las fecto lupa o el Font locator; potenciadores de sonido como Earbot e inclusive lectores de etiquetas para identificar correctamente los productos como Medbase.

\subsection{Salud}

Esta categoría constituye la de mayor entidad en la muestra de estudio, a la que pertencen tres de cada diez aplicaciones para senior (un total de 89 APPs). Dentro de Salud se incluyen diversos tipos de APPs que van desde la información para el desarrollo de una vida saludable, el aviso y dosificación de los medicamentos hasta la atención en el domicilio.

Para dar cabida a esta heterogenidad de aplicaciones se ha establecido diversas subcategorías de clasificación: Información de la salud, como Senior care compare o Info Quiz de Stall Geiatrics (sobre patologías vinculadas al sueño, incontinencia, etc.); alertas-recordatorio del estilo de TeleMed o MedsOnTime para la dosificación de medicamentos o iTriage para la agenda de citas médicas; APPs de vida saludable como las aplicaciones destinadas a promover el ejercicio físico y/o cognitivo como Smart Ageing; las de monitorización de la salud como Heart Ratting Monitoring o Care Zone, o las de cuidado en el hogar .

A esta última subcategoría pertencen las aplicaciones del estilo Elder 411 - Senior caregiving made easier with Doctor Marion de asistencia y cuidados de la salud en el hogar asi como otras como Wappa o Senior Care management, dirigidas al círculo pesonal personal de los mayores para efectuar un seguimiento de su salud en una suerte de red social de cuidados (familiares-cuidadores-sanitarios).

\subsection{Seguridad}

Una de las principales motivaciones en la adopción de la telefonía móvil por los usuarios plateados es la seguridad, finalidad principal del $13,6 \%$ de las APPs analizadas (un total de 39 unidades). El estudio desarrollado ha permitido señalar hasta tres subtipologías de aplicaciones vinculadas a la seguridad de las personas de edad: gestores de cámaras audovisiuales, que permiten a los familiares seguir el día a día de los mayores cuando están solos; emergencia, en la que se sitúan un estilo determinado de APPs como Be Safe, Gatts, Panic Button basados en un sistema sencillo y directo de interacción (en su mayoría con un único botón de grandes dimensiones) para alertar a familiares y profesionales del cuidado de una necesidad urgente de asistencia. Algunas de estas APPs como ManDown utilizan el propio sensor de movimiento del terminal para determinar la necesidad de asiastencia.

Finalmente, el tercer tipo de aplicaciones de seguridad son las de localización. Estas APPs se basan en la tecnología de posicionamiento de los smartphones para efectuar el segumiento o geolocalización de los mayores, especialmente de aquellos aquejados de algún tipo de demencia. En este sentido se han señaado dos tipologías de APPs, las que dan el posicionamiento del portador del teléfono en un mapa como Donde Está, y las que envía de forma automática un mensaje con la ubicación exacta $W R U$. Unas APPs que también se utilizan en la localización de los más jóvenes de la familia.

\subsection{Vida cotidiana}

En esta categoría se han clasificado todas aquellas aplicaciones móviles destinadas a facilitar o mejorar determinados aspectos de la vida cotidiana de las personas de edad, especialmente de aquellas que viven solas (28 APPs que constituyen en torno al $10 \%$ de la muestra). La mayor parte de las aplicaciones clasificadas en esta categoría ofrecen información de interés para los mayores como actividades sociales y culturales de su 
entorno como Waukesha Senior Calendar o Senior Living Communities, algunas de ellas son expansiones móviles de servicios online.

No obstante, resulta destacable un segundo tipo de APPs que se han etiquetado como organización personal. Esta subcategoría agrupoa al conjunto más heterogéneo de aplicaciones de la muestra de análisis: gestores de tareas (Senior friendly to do list), asistentes de compra y cocina (Recipes for senior), economia del hogar (Senior savings), etc.

\subsection{Entretenimiento}

Las oferta de entretenimiento agrupa el conjunto de APPs más numeroso de la muestra tras los contenidos de salud (52 unidades que constituyen el $18,4 \%$ de la muestra). Se trata de una serie de aplicaciones vinculadas al tiempo libre de los mayores como los juegos senior (Senior Solutions), revistas (FNV Senior, BoomerTimes) e inclusive APPs de turismo como Senior Tourism, y adaptadas expresamente a las necesidades y capacidades de las personas mayores.

Se trata de aplicaciones de juegos, revistas o turismo, creadas y adaptadas expresamente a las necesidades de las personas mayores. En algunos casos, como en el de los smart games, estas aplicaciones también tienen la finalidad de ejercitar capacidades cognitivas o motoras, por lo que se podría preveer una dupla catalogación de las mimas. No obstante, el entretenimiento constituye la principal finalidad de las mismas.

Dentro del apartado de entretenimiento, aunque encabalgado con el de comunicación e interacción social se han incluido las aplicaciones de citas. Unas APPs sencillas que tienen en común el acceso del individuo en una comunidad online para concer gente e incrementar sus lazos sociales. Estas aplicaciones tienen dos particularidades, el contacto individual aún dentro de una comunidad y sus destinatarios son el grupo de adultos maduros más que las franjas de mayor edad (tal como refleja su diseño y ergonomía).

\subsection{Comunicación interpersonal y redes sociales}

Además de las aplicaciones de accesibilidad, que facilitan y simplifican el acceso de las personas mayores a las funciones de conectividad y comunicación del teléfono, resulta preciso hablar de otra tipología de aplicaciones de interacción. Estas aplicaciones, que constituyen el 8,5\% de la muestra (24 aplicaciones), pueden ser servicios de comunicación personal como Toguethera o PreciouSatus o de interacción social como Tapestry o Easy Family Social.

La organizción de aplicaciones propuesta, al igual que sus categorías y subcategorías, ha procurado una clasificación lo más exahustiva posible aún partiendo del hecho de que la diversidad de funciones de algunas de las APPs analizadas pueden llevar a un etiquetado múltiples. Es el caso, por ejemplo, de You're onlune old once, Dr Sauss. una APP-Ebook interactiva que complementa la narración con otro tipo de contenidos como la información sanitaria o los juegos. En estos casos, se ha primado la función principal de la aplicación para su clasificación.

\section{APPS PARA LA COMUNICACIÓN PERSONAL Y LA INTERACCIÓN SOCIAL}

En lo que respecta a las aplicaciones diseñadas para la comunicación interpersonal y a la interacción social de los mayores, categoría a la que pertenecen 22 aplicaciones (dos de ellas con presencia en iTunes y Google Play), se ha efectuado una subclasificación atendiendo a sus funcionalidades y al tipo de comunicacióninteracción que permiten.

A partir de estos criterios se han establecido tres categorías de aplicaciones : mensajería instantánea, redes sociales específicas y APPs para incrementar la accesibilidad de plataformas sociales ya existentes.

El éxito recabado por las aplicaciones de mensajería instantánea como WhatsApp y Line, al igual que de otros servicios similares como Skype, ha tenido su reflejo en las aplicaciones de comunicación para seniors. Una de cada dos aplicaciones de interacción-comunicación (11 APPs) permiten a los mayores comunicarse 
directamente con uno de los contactos de su red a través de texto (Printogram), imágenes o pictogramas (Treeo), audio (Talkly), videollamadas (VissBuzz) o mensajes multimedia (Cubigo, Óscar Junior, Toghethera, Unus tactus lite). Alguna de estas aplicaciones, como Sentab, Facelook o Are you OK se ha diseñado también para la interacción con profesionales de la salud.

Las aplicaciones de redes sociales, subcategoría a la que pertenecen 8 de las APPS de la muestra, permiten que los mayores interactúen de forma pública con su comunidad social. Además de simplificar dicha interacción estas aplicaciones tienen en común el carácter restringido de sus comunidades, en su mayoría familiares, que garante la privacidad de sus miembros.

Estas redes sociales son Tapestry, PreciouStatus, Post55 (generalistas), Cura y Active Seniors (comunidades de apoyo y de e-learning, respectivamente). Entre dichas APPs resulta destacable el peso de redes sociales como Checkin'in, FamilyFul, Familonet y FamyliFul que incorporan información sobre la ubicación del usuario en sus actualizaciones de estado, con el consecuente incremento en su seguridad.

Finalmente las aplicaciones de accesibilidad a plataformas sociales, subcategoría a la que pertenecen Facebook para mayores, Easy Family Social (para Facebook) y Simple Twitter, tienen como cometido facilitar la interacción de los usuarios plateados en dichas redes sociales mayoritarias. Estas APPs simplifican el acceso a las principales funciones de la plataforma a través de interfaces sencillas, de diseño ergonómico, más accesibles para las personas que las aplicaciones oficiales de dichas plataformas.

Precisamente accesibilidad y simplicidad constituyen las características comunes de las aplicaciones analizadas. Unas características que pueden ser clave en la adopción de estas aplicaciones por los usuarios de mayor edad y, por ende, en el beneficio de sus potencialidades de cara a un envejecimiento activo.

\section{CONCLUSIONES}

La tecnología móvil presenta grandes potencialidades en el marco del envejecimiento activo. La percepción de seguridad, ligada a estos dispositivos, y sus posibilidades de personalización constituyen una oportunidad para limitar la brecha digital y lograr una sociedad para todas las edades.

Los smartphones cada vez más presentes en los bolsillos de los mayores, y con éstos a un universo de aplicaciones y funcionalidades que pueden mejorar su calidad de vida en el sentido más amplio.

Atendiendo a su finalidad estas aplicaciones se han clasificado en accesibilidad, salud, seguridad, vida cotidiana, entretenimiento y comunicación interpersonal y redes sociales. Unas aplicaciones que tienen en común contar con una mayor accesibilidad y ergonomía que las APPs convencionales.

En efecto, en el caso de las aplicaciones de salud cuyos destinatarios son familiares y profesionales el diseño, contenidos y navegación del interfaz son más complejos e -inclusive- crípticos para los individuos objeto del cuidado.

La relevancia de las redes e interacciones sociales para el desarrollo personal, la inclusión y la participación, ha llevado al desarrollo de diversas aplicaciones móviles que multiplican las posibilidades de comunicación y conexión de los mayores con su entorno. Tomando como referencia el tipo de interacción (individual o social) y su funcionalidad se puede hablar de APPs de mensajería instantánea, de redes sociales o de accesibilidad de redes sociales. Estas últimas categorías suscitan una reflexión en torno a dos conceptos implícitos en el envejecimiento activo: inclusión y privacidad.

El desarrollo de gestores de redes sociales específicos para las personas de edad y su entorno implica una mayor adaptación de estas aplicaciones a las necesidades y capacidades de los mayores, estimulando su participación e inclusión. El carácter restringido de estas APPs y de sus comunidades supone una oportunidad tanto para el establecimiento de redes sociales sólidas como para mantener la seguridad y privacidad de sus miembros. 
Las APPs de redes sociales senior, no obstante, presentan una importante limitación: la propia comunidad social. Pese a que dichas plataformas son totalmente accesibles para las personas mayores su uso, en detrimento de otros servicios de mayor penetración como Facebook o Twitter, crea un espacio social paralelo con consecuencias negativas en términos de e-inclusión.

Si se parte del hecho de que las redes sociales las conforman las personas y sus interacciones, no las plataformas, se considera más interesante el desarrollo de APPs destinadas a mejorar la accesibilidad de las plataformas sociales con mayor número de seguidores. En las aplicaciones de redes sociales senior las interacciones obedecen al encuentro entre mayores y familiares, no a una actividad cotidiana de socialización en el sentido más amplio.

En este sentido, además de la mayor accesibilidad de las plataformas generalistas, se propone el desarrollo de programas de educación digital que incidan en la seguridad y privacidad en Internet, de cara a mejorar la einclusión de los mayores.

\section{REFERENCIAS BIBLIOGRÁFICAS Y HEMEROGRÁFICAS}

ABASCAL, J.; CIVIT, A. (2000): Mobile communication for people with disabilities and older people: New opportunities for autonomous life. En Proceedings of the 6th ERCIM Workshop. Florencia: ERCIM, pp. 255-268 [en línea]. [Consulta: 10 marzo 2015]. < http://goo.gl/cHG9KX >.

AGUDO, S.; PASCUAL, M.A.; FOMBONA, J. (2012): Usos de las herramientas digitales entre las personas mayores. Comunicar, vol. 39, n² 29, pp.193-201.

ALDANA, G.; FONSECA, C.: GARCÍA, L. (2013): El significado de la vejez y su relación con la salud en ancianas y ancianos integrados a un programa de envejecimiento activo. Revista Digital Universitaria, vol.14, $\mathrm{n}^{\circ}$ 4 [en línea]. [Consulta: 10 marzo 2015]. < http://goo.gl/VPgGLc >.

AMICHAI-HAMBURGER, Y.; BEN-ARTZI, E. (2003): Loneliness and Internet use. Computers in human behavior, vol. 19, n 1, pp.71-80.

BEAUVOIR, S. de (1983): La vejez. Barcelona: Edhasa.

BELL, C.; FAUSSET, C.; FARMER, S.; NGUYEN, J.; HARLEY, L.; FAIN, W. B. (2013). Examining social media use among older adults. En Proceedings of the 24th ACM Conference on Hypertext and Social Media. New York: AMC (pp. 158-163).

BLAKE, H. (2008): Innovation in practice: mobile phone technology in patient care. British journal of community nursing, vol.13, n4, pp.160-165.

BURKHARD, M.; KOCH, M. (2012): Social Interaction Screen. Making Social Networking Services Accessible for Elderly People. i-com Zeitschrift für interaktive und kooperative Medien, vol. 11, n³, pp. 3-7. [en línea].

[Consulta: 10 marzo 2015]. < http://goo.gl/83QE3s>.

CAGLEY, M.; LEE, M. (2009): Social support, networks, and happiness. Today's Research on Aging: Program and Policy Implications, 17. Washington: Population Reference Bureau.

CHARNESS, N. (2014): Utilizing technology to improve older adult health. In Occupational therapy in health care, 28(1), 21-30.

GAO, J.; KORONIOS, A. (2010): Mobile Application Development for Senior Citizens. [en línea]. [Consulta: 10 marzo 2015]. < http://goo.gl/nwnpCZ>.

CHARNESS, N.; PARKS, D. C.; SABEL, B. A. (2001): Communication, technology and aging: Opportunities and challenges for the future. New York: Springer Publishing Company. 
COELHO, J.; DUARTE, C. \& AL. (2012): Involving All Stakeholders in the Development of TV Applications for Elderly. ThinkMind. International Journal On Advances in Intelligent Systems, vol. 5, n 3-4. [en línea]. [Consulta: 10 marzo 2015]. <http://goo.gl/Hr2Cdm >.

CONCI, M. (2007): Mobile Phone Acceptance by Older People. Building and Validation of a theoretical model. Tesis doctoral. Trento: Universita Degli Studi di Trento.

CONDE, M. Á., GARCÍA-PEÑALVO, F. J., Y MATELLÁN-OLIVERA, V. (2014): Mobile apps repository for older people. En Proceedings of the Second International Conference on Technological Ecosystems for Enhancing Multiculturality. New York: ACM. (pp. 725-731).

CORNEJO, R.;TENTORI, M; FAVELA, J. (2013): Enriching in-person encounters through social media: A study on family connectedness for the elderly. Journal of Human-Computer Studies, nº 71, pp. 889-899.

COTTEN, S. R. (2011): Overcoming digital divides in assisted and independent living communities: Results from a randomized trial. Gerontologist, $\mathrm{n}^{\circ} 51$, pp. 220-230.

CZAJA, S.; LEE, C. (2007): The impact of aging on access to technology. Universal Access in the Information Society, vol.5, $\mathrm{n}^{\circ} 4$, pp. 341-349.

DEL CAMPO, A.; LIMA MAESTRO, R. D. (2014): Análisis crítico del discurso publicitario institucional/comercial sobre las personas mayores en España. Comunicar, vol. 42, n² 20, pp. 189-197.

DROBICS, M; SMITH, S. (2014): Game-Based IT Solutions for Active and Healthy Aging. En GOBEL, S.; WIEMEYER, J. (Eds.), Games for Training, Education, Health and Sports. 4th International Conference on Serious Games. Darmstadt: Springer International Publishing. (pp. 8-11).

FERCGLA, J. E. (1992): Una antropología de la ancianidad. Barcelona: Anthropos.

FILE, T. (2013): Computer and internet use in the United States. Population Characteristics. [en línea]. [Consulta: 10 marzo 2015]. <http://www.census.gov/prod/2013pubs/p20- 569.pdf >.

GAO, J.; KORONIOS, A. (2010): Mobile application development for senior citizens. [en línea]. [Consulta: 10 marzo 2015]. <http://www.pacis-net.org/file/2010/S05-03.pdf>.

GIRÓ, J. (2004): El significado de la vejez. In Autor (Coord.), Envejecimiento y sociedad. Una perspectiva pluridisciplinar. Logroño: Universidad de la Rioja. (pp.19-46).

HAMPTON, K. N.; GUPTA, N. (2008): Community and social interaction in the wireless city: wi-fi use in public and semi-public spaces. New Media \& Society, vol. 10, n 6, pp. 831-850.

HARRINGTON, T. L.; HARRINGTON, M. K. (2000): Gerontechnology: Why and how. The Netherlands: Shaker KURNIAWAN, S. (2008): Older people and mobile phones: A multi-method investigation. International Journal of Human-Computer Studies, vol. 66, n²12, pp. 889-901.

LEHR, U. (1980): Psicología de la senectud. Barcelona: Herder.

LING, R. (2008): Exclusion or self-isolation? Texting and the elderly users. I The information society, vol. 24, $\mathrm{n}^{\circ}$ 5, pp. 334-341.

MARTÍNEZ, R.; CABECINHAS, R.; LOSCERTALES, F. (2011): Mayores universitarios en la Red. Comunicar, vol. $37, n^{\circ} 20$, pp. 89-95.

MCGEE-LENNON, M. R. (2008): Requirements engineering for home care technology. In Proceedings of the SIGCHI Conference on Human Factors in Computing Systems. Ney York: ACM (pp. 1439-1442).

OKSMAN, V. (2006): Young people and seniors in Finnish 'mobile information society'. Journal of Interactive Media in Education, vol. 2, nº 1. [en línea]. [Consulta: 10 marzo 2015]. 
<http://jime.open.ac.uk/articles/10.5334/2006-3/>.

PEDELL, S.; BEH, J.; MOZUNA, K.; DUONG, S. (2013): Engaging older adults in activity group settings playing games on touch tablets. In Proceedings of the 25th Australian Computer-Human Interaction Conference: Augmentation, Application, Innovation, Collaboration. New York: ACM. (pp. 477-480).

PEW RESEARCH CENTER (2014): Social networking fact sheet. [en línea]. [Consulta: 10 marzo 2015]. < http://www.pewinternet.org/fact-sheets/social-networking-fact-sheet/ (12-10-2014).

PIRIYAPOKANON, T (2011): Is a Big Button Interface Enough for Elderly Users?: Towards user interface guidelines for elderly users. [en línea]. [Consulta: 10 marzo 2015]. <

http://www.idt.mdh.se/examensarbete/index.php?choice=show\&id=1091>

PLAZA, I.; MARTÍN, L.; MARTIN, S; MEDRANO, C. (2011): Mobile applications in an aging society: Status and trends. Journal of Systems and Software, vol. 84, $\mathrm{n}^{\circ}$ 11, pp.1977-1988.

PUTNAM, R.D. (1995): Bowling Alone: America's Declining Social Capital. I Journal of Democracy, vol. 6, pp. 6578.

PARALES, C. J.; DULCEY, E. (2002): La construccion social del envejecimeinto y de la vejez: un analisis discursivo en prensa escrita. Revista Latinoamericana de Psicología vol. 34, n 1/2, pp.107-121.

SÁNCHEZ, M.; HATTON-YEO, A. (2012): Active Ageing and Intergenerational Solidarity in Europe: A Conceptual Reappraisal from a Critical Perspective. Journal of Intergenerational Relationships, vol. 10, $\mathrm{n}^{\circ}$ 3, pp. 276-293.

VALENZUELA, S.; PARK, N.; KEE, K. F. (2008): Lessons from Facebook: The effect of social network sites on college students' social capital. En 9th International Symposium on Online Journalism. Austin: Texas. [en línea]. [Consulta: 10 marzo 2015]. <http://goo.gl/ZY1pgy>

ZHANG, S.; JIANG, H.; CARROLL, J. M. (2011): Integrating online and offline community through Facebook. En Collaboration Technologies and Systems (CTS), 2011 International Conference on Technologies and systems. Philadelphia: IEEE (pp. 569-578).

SLEGERS, K.; VAN BOXTEL, M.; JOLLES, J. (2009): Effects of computer training and internet usage on cognitive abilities in older adults: a randomized controlled study. Aging clinical and experimental research, vol. 21, n¹, pp. 43-54.

VILTE, D.; SALDADAÑO, V. ; MARTíN, A. ; GAETÁN, G. (2013): Evaluación del uso de redes sociales en la tercera edad. [en línea]. [Consulta: 10 marzo 2015]. <http://goo.gl/UhrGHY>.

WERNER, C. A. (2011): The older generation: 2010. U.S. Census Bureau. [en línea]. [Consulta: 10 marzo 2015]. $<\mathrm{http}: / / g o o . g l / 8 q O a p U>$.

WILLIAMS, D.; AHAMED, S. I.; CHU, W. (2014): Designing Interpersonal Communication Software for the Abilities of Elderly Users. En Computer Software and Applications Conference Workshops. IEEE 38th International .Vasteras: IEEE (pp. 282-287).

YU, P.; LI, H.;GAGNON, M. P. (2009): Health IT acceptance factors in long-term care facilities: a cross-sectional survey. International Journal of Medical Informatics, vol. 78, n 4, pp. 219-229.

ZHOU, J.; PEI-LUEN, P. R.; SALVENDY, G. (2014): Older adults text entry on smartphones and tablets. Investigating effects of display size and imput method on aceptance and performance. International Journal of Human Computer Interaction, vol. 30, $\mathrm{n}^{\circ}$ 9, pp. 727-739. 
Xabier Martínez-Rolán es licenciado en Comunicación Audiovisual por la Universidade de Vigo y profesor asociado en la misma Universidad, donde imparte asignaturas relacionadas con la comunicación digital y los dispositivos móviles. Ha orientado su investigación hacia la comunicación en redes sociales, nuevos soportes publicitarios y comunicación alternativa, ámbitos en los que también desarrolla su labor profesional.

Teresa Piñeiro-Otero, es doctora en Comunicación por la Universidade de Vigo y Profesora Ayudante Doctora en la Universidade da Coruña. Miembro del grupo de investigación "Comunicación y Cultura Interactiva" ha orientado su investigación hacia las nuevas manifestaciones mediáticas en el contexto de la convergencia, con especial atención a los dispositivos móviles.

Ámbitos. Revista Internacional de Comunicación, n.29, año 2015, tercer trimestre (verano). Recibido: 01/03/2015

Aprobado: 16/06/2015 